\title{
A Nasogastric Tube Inserted into the Gastrocutaneous Fistula
}

\author{
Yang Soo Kim, M.D., Joon Sung Kim, M.D., In Hee Yu, M.D., Ji Young Jeong, M.D., \\ Sung Hee Jung, M.D., Yil Ryun Jo, M.D., Myung Eun Chung, M.D.
}

Department of Rehabilitation Medicine, College of Medicine, The Catholic University of Korea, Seoul 130-709, Korea

We reported a case in which a nasogastric tube was inserted into the gastrocutaneous fistula, diagnosed by abdominal computed tomography. A 78-year-old man with a history of recurrent cerebral hemorrhage had a percutaneous endoscopic gastrostomy tube due to dysphagia for 2 years. However, soft tissue infection at the gastrostomy site caused the removal of the tube. Immediately, antibiotic agents were infused. For appropriate hydration and medication, a nasogastric tube was inserted. However, there was no significant improvement of the soft tissue infection. Moreover, the amount of bloody exudate increased. Abdominal computed tomography revealed the nasogastric tube placed under the patient's skin via gastrocutaneous fistula. The nasogastric tube was removed, and an antibiotic agents were maintained. After 3 weeks, the signs of infection fully improved, and percutaneous endoscopic gastrostomy was performed again. This case shows necessities of an appropriate interval between removal of the gastrostomy tube and insertion of a nasogastric tube, and suspicion of existence of gastrocutaneous fistula.

Key Words Gastrocutaneous fistula, Percutaneous endoscopic gastrostomy, Nasogastric tube

\section{INTRODUCTION}

Dysphagia from stroke can cause delays in the patient functional outcomes, and is reported as the primary cause of various medical complications such as aspiration pneumonia, affecting approximately $25-45 \%$ of

Received September 8, 2010; Accepted December 20, 2010

Corresponding author: Myung Eun Chung

Department of Rehabilitation Medicine, St. Paul's Hospital, College of Medicine, The Catholic University of Korea, 620-56, Jeonnong 1-dong, Dongdaemun-gu, Seoul 130-709, Korea

Tel: +82-2-958-2307, Fax: +82-2-958-2089, E-mail: coltrane@catholic. ac.kr

(c) This is an open-access article distributed under the terms of the Creative Commons Attribution Non-Commercial License (http:// creativecommons.org/licenses/by-nc/3.0) which permits unrestricted noncommercial use, distribution, and reproduction in any medium, provided the original work is properly cited.

Copyright $\odot 2011$ by Korean Academy of Rehabilitation Medicine patients. ${ }^{1}$ Penington et al. also reported that subclinical laryngeal aspiration occurs in $40-60 \%$ of patients with dysphagic stroke. $^{2}$

In patients with dysphagic stoke, nasogastric tube feeding is comparatively easy for nutritional support in initial stroke, can incur the patient uneasiness, is prone to being dislodged, and can cause aspiration pneumonia from mechanical obstruction. Percutaneous endoscopic gastrostomy, on the other hand, is comparatively simple to perform, has fewer side effects, and provides cosmetic advantage. It is thus recommended for patients with longterm dysphagic stroke to have percutaneous endoscopic gastrostomy if there is no gastrointestinal lesion. ${ }^{3}$

However, when performing percutaneous endoscopic gastrostomy, side effects can occur such as bleeding, gastric ulcer, intestinal perforation, or penetration 
through the left lobe of the liver. Besides, it is inevitable that it needs to be removed after the operation for various reasons, most commonly infection. According to Pien et al., $4.8 \%$ of patients had infection after percutaneous endoscopic gastrostomy. ${ }^{4}$ In cases of infection caused by gastrostomy tube, the tube was usually removed and antibiotic therapy was applied, and a nasogastric tube was inserted for nutritional support, hydration and smooth drug administration. We report the first case in which for a patient with long-term nutrition via gastrostomy tube, terminal portion of nasogastric tube was inserted through gastrocutaneous fistula after removal of the gastrostomy tube due to infection.

\section{CASE REPORT}

A 78-year-old male patient was diagnosed as hypertension and on medication came to our hospital after having a hematoma evacuation in an American hospital because of left thalamic hemorrhage and right hemiplegia. In the computed tomography (CT) of his brain after hospitalization, intracerebral hemorrhage in the left frontal lobe and intraventricular hemorrhage were discovered. This led to operating hematoma evacuation, and a month later, ventriculoperitoneal shunt was installed because of hydrocephalus. Two months later, he was transferred to the rehabilitation medicine department for comprehensive rehabilitation. In the neurological examination at the time of transfer, his conscious state was maintained, but conversation was impossible and verbal responses were not observed due to the tracheostomy. He was already diagnosed as dysphagia at the time of hospitalization in the U.S. and had percutaneous endoscopic gastrostomy. When he was transferred to our hospital, he was still having nutrition support through the percutaneous gastrostomic tube. A month later, soft tissue infection occurred around the tube, so ceftriaxone was applied for antibiotic therapy, and the tube was replaced after the inflammation was relieved while the existing passage of gastrostomic tube was maintained. In the videofluoroscopic swallowing study carried out a month later, aspiration was still found so the gastrostomic tube was maintained, which led to the same result when the test was carried out three months later. Due to the malfunction of the gastrostomic tube a month later, the tube was replaced with the

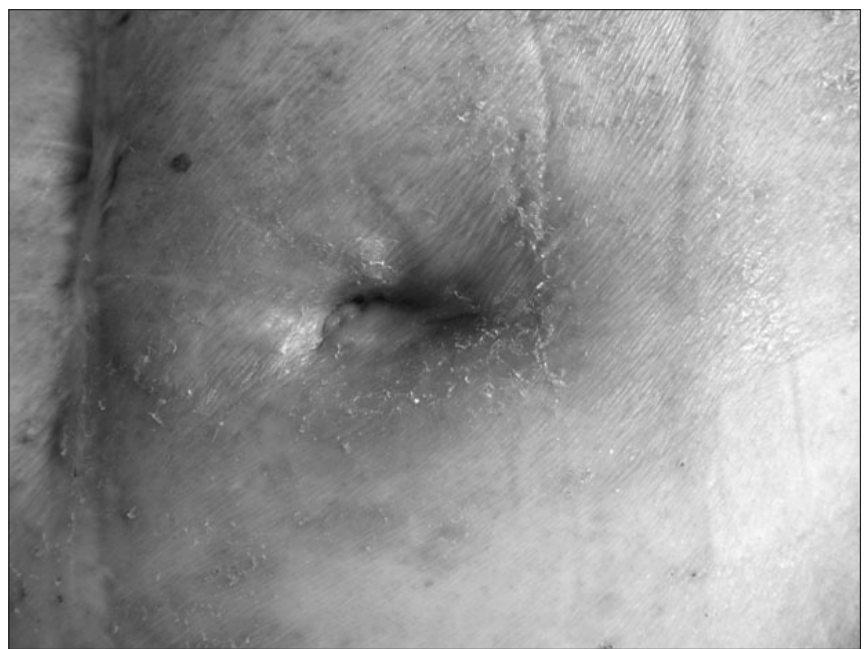

Fig. 1. Erythematous skin is observed in the gastrostomy site after removal of tube.

passage maintained, but was inserted again because the tube was dislodged during sleep two weeks after the replacement. In the videofluoroscopic swallowing study carried out 4 months later, aspiration still continued with decreased amount, and intermittent spontaneous swallowing activities were observed. However, we decided to maintain the tube and replaced it due to dysfunction. Three months after the replacement, the erythema and exudate containing blood clots were observed on the skin around the tube insertion (Fig. 1). In the blood test, the infectious signs were found with white blood cells $12,700 /$ ul, segment neutrophils $76.6 \%$, erythrocyte sedimentation rate $73 \mathrm{~mm} / \mathrm{h}$, and C-reactive protein $17.12 \mathrm{mg} / \mathrm{dl}$, while body temperature was maintained under $37^{\circ} \mathrm{C}$. Urinalysis and urinary chemistry was not specific, bowel sounds was normal, but abdominal tenderness and rebound tenderness could not be examined due to the patient's cognitive dysfunction. No anomalies were seen in the plain X-ray of chest and abdomen. Since infection in the gastrostomic tube insertion site was suspected, the tube was removed after microbiological culture. Ceftriaxone was applied for the purpose of empirical antibiotic therapy, and peripheral parenteral nutrition was initiated. The nasogastric tube was inserted the next day for the medication of hypertension, and was confirmed to have been located at the epigastrium by auscultation. The nasogastric tube was used only for medication. Ceftriaxone was maintained because Klebsiella pneumoniae sensitive to $\beta$-Lactam 

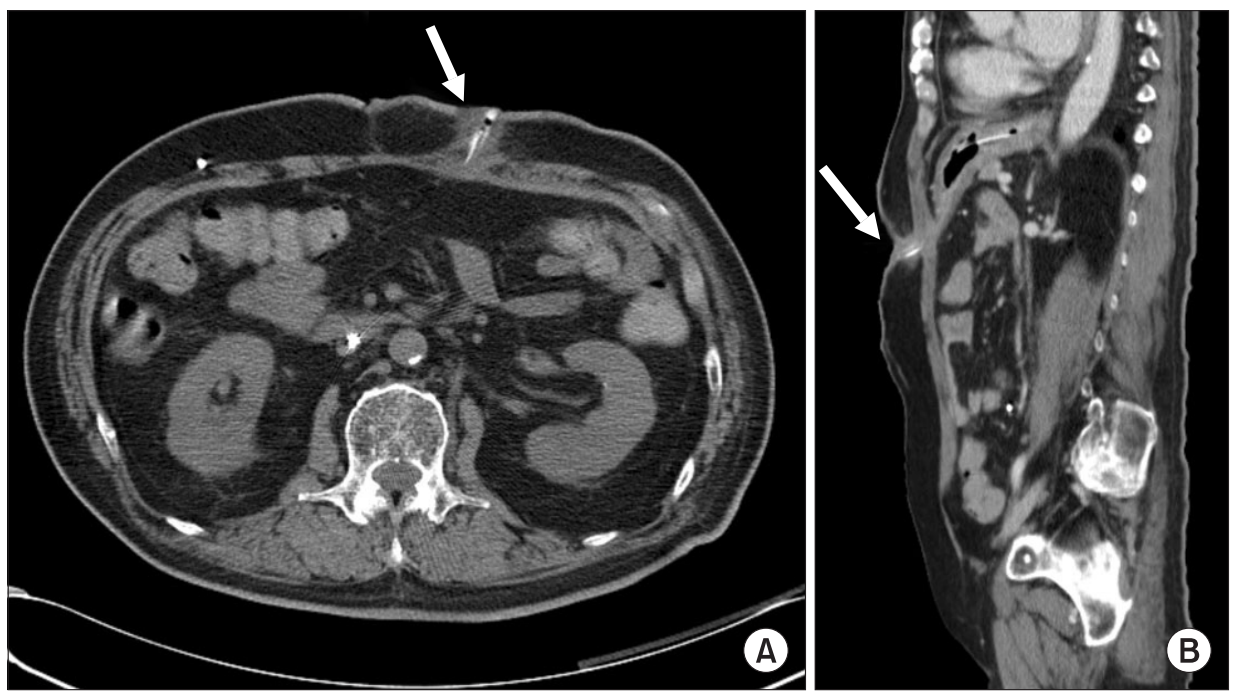

Fig. 2. CT findings show a transverse cut (A) and a coronal cut (B) of nasogastric tube placed beneath the skin via gastrocutaneous fistula.

antibiotics was identified. Three days after removing the percutaneous gastrostomic tube and starting antibiotic therapy, the blood test became normal, but the erythema around the part of the tube insertion and the exudate containing blood clot did not improve. Since complex infection of anaerobes was suspected, metronidazole was orally administered. At the same time, CT was performed 4 days after the nasogastric tube insertion because of possible subcutaneous and intraperitoneal abscess. The abscess was not found by CT, but the terminal portion of the nasogastric tube penetrated the gastric mucous membrane and was located beneath the skin through the gastrocutaneous fistula with soft tissue infection around the gastrocutaneous fistula (Fig. 2). We thus diagnosed it as skin infection by the mislocation of the terminal portion of the nasogastric tube, removed the tube, administered $2 \mathrm{~g}$ of ceftriaxone and 1,500 mg of metronidazole daily, and inserted a central venous catheter for the purpose of total parenteral nutrition. Twenty days later, the skin around the removed gastrostomic tube was found to be normal, blood test was normal, and no indurate nodules were observed. Percutaneous endoscopic gastrostomy was thus performed on the spot $5 \mathrm{~cm}$ away from where the previous gastrostomic tube was inserted. No additional complication was observed.

\section{DISCUSSION}

Even though nasogastric tube insertion is a comparatively easy operation, various problems can occur such as esophageal laceration during insertion, mislocation, metabolic disorder, and dehydration. After nasogastric tube insertion, there are many ways to confirm the location of the terminal portion: to make the patient use their voice, to perform suction of gastric content, to auscultate air bubbles, and to perform plain chest $\mathrm{X}$-ray. Neither of these, however, can verify the correct location of the nasogastric tube inside of the stomach wall. ${ }^{5}$ Among mislocations of the nasogastric tube, the intubation to the respiratory system is most frequent because the terminal tube cannot be inspected by eye during insertion. Most cases are intubation to the trachea, but some reports are on pneumothorax through the lungs. ${ }^{6}$ Some are a large quantity of aspiration caused by the terminal tube located in the pharyngeal airway and esophagus, or within the intracranial penetration through the ethmoid bone. Wynne et al. also reported a nasogastric tube located in the Eustachian canal. ${ }^{5,7}$

After performing percutaneous endoscopic gastrostomy, it is common for it to be placed for a long time unless there is any complication like infection or tubal dysfunction. However, in case of removing a gastrostomic tube, gastrocutaneous fistula can remain, which is naturally closed after a few hours or days. Therefore, $2-44 \%$ of patients with gastrocutaneous fistula are reported to remain for more than a week. It mostly occurs when the tube is placed for as long as 9-11 months, and is also related to delayed stomach emptying, foreign body within the stomach such as silk suture, chronic granulomatous disease or Crohn's disease. ${ }^{8}$ Some reports show gastrocutaneous fistula continuing a month after 
removing the gastrostomic tube. Kobak et al. reported that it was closed at 53\% using argon plasma coagulation and $\mathrm{H} 2$ antagonist. ${ }^{9}$ Therapeutic endoscopy could also be applied, with the defect of the tissue sutured using the clip. $^{10}$

Patients with percutaneous gastrostomic tubes often need to remove the tube because of infection or accidents. In many cases, nasogastric tubes are inserted for orally administered medication, whereas short-term nutrition supply can be through the vessel. However, it cannot be applied clinically in general to delaying the nasogastric tube insertion bearing in mind the existing gastrocutaenous fistula, so nasogastric tube is usually inserted 24 hours after removing the gastrostomic tube when there is no particular problem. In this case report, the nasogastric tube was also inserted one day after removing the gastrostomic tube, in order to confirm its location, auscultation and suction of gastric contents. However, we could not find evidence of the nasogastric tube invading the gastrocutaenous fistula. Even though confirming the nasogastric tube through abdomen X-ray tells the running status within the alimentary canal, gastrocutaenous fistula usually occurs vertically to the direction of the alimentary canal, making it difficult to confirm the locations of the nasogastric tube and gastrocutaneous fistula by two-dimensional X-ray. In addition, through lateral abdominal plain $\mathrm{x}$-ray, it is also difficult to catch the shade of the terminal portion of the nasogastric tube because of abdominal organs. Considering that the terminal nasogastric tube is observed to be located in gastrocutaenous fistula with soft tissue infection 4 days after the insertion, the possible transition of the tube from gastric fundus to gastrocutaneous fistula by peristalsis cannot be excluded. The gastrostomic tube needs to be removed after percutaneous endoscopic gastrostomy for many reasons. This is particularly the case when it is removed because of infection, secretion and erythema are involved where the tube is removed, making it hard to precisely estimate the existence of gastrocutaneous fistula. Moreover, when the nasogastric tube is inserted into gastrocutaneous fistula, its location cannot be examined exactly by simple physical examination or chest plain X-ray. The case of the nasogastric tube locating in the gastrocutaneous fistula and causing soft tissue infection is not common. It is important to check the existence of remaining gastrocutaneous fistula, in case there is no improvement in spite of proper antibiotic therapy as seen in this case study. It is also important to closely observe the existence of gastrocutaneous fistula when removing the percutaneous gastrostomic tube and inserting the nasogastric tube.

\section{REFERENCES}

1. Veis SL, Longemann JA. Swallowing disorders in persons with cerebrovascular accident. Arch Phys Med Rehabil 1985; 66: 372-375

2. Penington GR, Krutsch JA. Swallowing disorders: assessment and rehabilitation. Br J Hosp Med 1990; 44: 17-22

3. Raha SK, Woodhouse KW. Who should have a PEG? Age Ageing 1993; 22: 313-315

4. Pien EC, Hume KE, Pien FD. Gastrostomy tube infections in a community hospital. Am J Infect Control 1996; 24: 353-358

5. Wynne DM, Borg HK, Geddes NK, Fredericks B. Nasogastric tube misplacement into Eustachian tube. Int J Pediatr Otorhinolaryngol 2003; 67: 185-187

6. Sorokin R, Gottlieb JE. Enhancing patient safety during feeding-tube insertion: a review of more than 2000 insertions. J Parenter Enter Nutr 2006; 30: 440445

7. Rahimi-Movaghar V, Boroojeny SB, Moghtaderi A, Keshimirian B. Intracranial placement of a nasogastric tube. A lesson to be re-learnt? Acta Neurochir (Wien) 2005; 147: 573-574

8. Aronian JM, Redo SF. Gastrocutaneous fistula after tube gastrostomy. Incidence in infants and children. N Y State J Med 1974; 74: 2364-2366

9. Kobak GE, McClenathan DT, Schurman SJ. Complications of removing percutaneous endoscopic gastrostomy tubes in children. J Pediatr Gastroenterol Nutr 2000; 30: 404-407

10. Siddiqui AA, Kowalski T, Cohen S. Closure of a nonhealing gastrocutaneous fistula using an endoscopic clip. South Med J 2007; 100: 75-76 